EPJ Web of Conferences 81, 01018 (2014)

DOI: $10.1051 /$ epjconf/ 20148101018

(C) Owned by the authors, published by EDP Sciences, 2014

\title{
In-medium $\bar{K}$ interactions and bound states
}

\author{
Avraham Gal ${ }^{1}$, a , Eli Friedman ${ }^{1}$, Nir Barnea ${ }^{1}$, Aleš Cieplý ${ }^{2}$, Jiří Mareš², and Daniel Gazda ${ }^{3}$ \\ ${ }^{1}$ Racah Institute of Physics, The Hebrew University, Jerusalem 91904, Israel \\ ${ }^{2}$ Nuclear Physics Institute, 25068 Řež, Czech Republic \\ ${ }^{3} E_{C T}{ }^{*}$, Villa Tambosi, I-38123 Villazzano (Trento), Italy
}

\begin{abstract}
Correct treatment of subthreshold $\bar{K} N$ dynamics is mandatory in $K^{-}$-atom and $\bar{K}$-nuclear bound-state calculations, as demonstrated by using in-medium chirally-based models of $\bar{K} N$ interactions. Recent studies of kaonic atom data reveal appreciable multinucleon contributions. $\bar{K}$-nuclear widths larger than $50 \mathrm{MeV}$ are anticipated.
\end{abstract}

\section{Introduction}

The $\bar{K} N$ interaction near and below threshold is attractive in models that generate dynamically the subthreshold $s$-wave resonance $\Lambda(1405)$, providing sound motivation to search for $K^{-}$bound states in nuclei [1]. Subthreshold $K^{-} N$ scattering amplitudes are needed for calculating such states, even in kaonic atoms for which the kaon energy is essentially at threshold [2-4]. However, subthreshold $\bar{K} N$ scattering amplitudes are highly model dependent, as demonstrated in Fig. 1 showing that two distinct NLO chiral-model fits to scattering and reaction data above and at threshold could generate $K^{-} p$ scattering amplitudes differing substantially from each other in the subthreshold region. Fit-I amplitude, nevertheless, is quite similar to the NLO amplitudes derived by Ikeda, Hyodo and Weise (IHW) [7] and by Cieplý and Smejkal (CS) [8], both of which were used in our recent calculations.

The present review follows Refs. [9, 10], highlighting recent progress made by the PragueJerusalem Collaboration towards incorporating medium modifications, particularly those implied by the energy dependence of $K^{-} N$ scattering amplitudes [11-16]. In-medium $\bar{K} N$ scattering amplitudes are discussed in Sect. 2, focusing on the connection between their (subthreshold) energy and density dependencies. The use of such in-medium $K^{-} N$ scattering amplitudes in kaonic-atom calculations and fits is discussed in Sect. 3. Related applications to kaonic bound-state calculations are discussed in Sect. 4 for few-body systems, and in Sect. 5 for many-body systems. A brief summary and outlook in Sect. 6 concludes this presentation.

\section{In-medium amplitudes and energy versus density dependence}

Here we follow Cieplý and Smejkal [8] who introduced meson-baryon coupled-channel energydependent separable $s$-wave interactions matched to $\mathrm{SU}(3)$ scattering amplitudes in up to next-toleading order (NLO) of the chiral expansion for strangeness -1 . It is gratifying that the TomozawaWeinberg LO term provides fair approximation to the corresponding $\bar{K} N$ amplitudes [7, 8]. Solving

a. e-mail: avragal@savion.huji.ac.il 

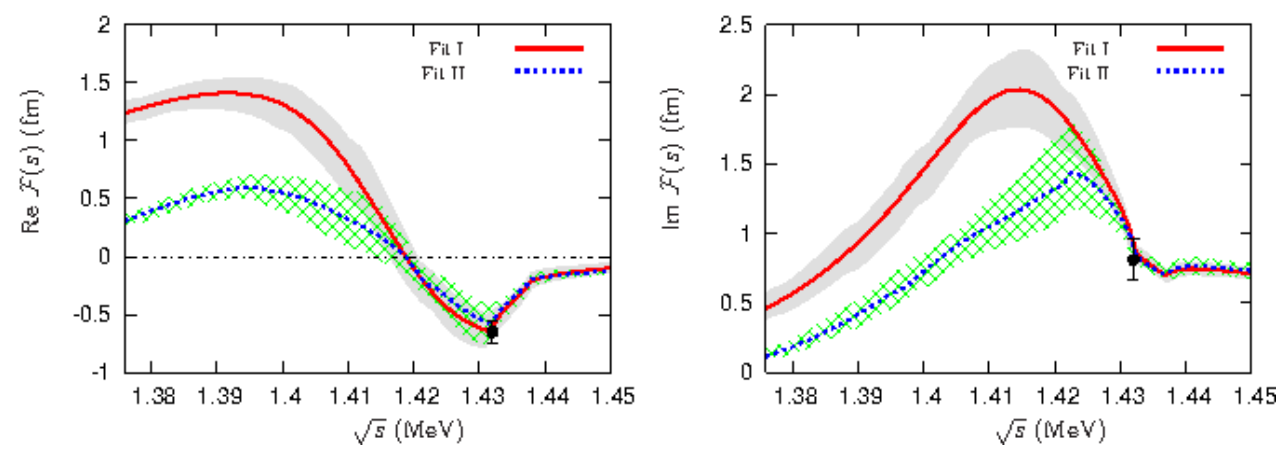

Figure 1. Real (left panel) and imaginary (right panel) parts of the $K^{-} p$ center-of-mass (cm) scattering amplitudes generated in two NLO chiral-model fits [5]. The $K^{-} p$ threshold values marked by solid dots follow from the SIDDHARTA measurement of kaonic hydrogen $1 s$ level shift and width [6]. Figure adapted from Ref. [5].

the in-medium coupled-channel Lippmann-Schwinger equations $F=V+V G F$ with these potential kernels leads to a separable form of in-medium scattering amplitudes $F_{i j}$, given in the two-body cm system by

$$
F_{i j}\left(k, k^{\prime} ; \sqrt{s}, \rho\right)=g_{i}\left(k^{2}\right) f_{i j}(\sqrt{s}, \rho) g_{j}\left(k^{\prime 2}\right),
$$

with momentum-space form factors $g_{j}\left(k^{2}\right)$, where $j$ runs over channels, and in-medium reduced amplitudes $f_{i j}(\sqrt{s}, \rho)$ expressed as

$$
f_{i j}(\sqrt{s}, \rho)=\left[(1-v(\sqrt{s}) \cdot G(\sqrt{s}, \rho))^{-1} \cdot v(\sqrt{s})\right]_{i j} .
$$

Here, $G$ is a channel-diagonal Green's function in the nuclear medium:

$$
G_{n}(\sqrt{s}, \rho)=-4 \pi \int_{\Omega_{n}(\rho)} \frac{d^{3} p}{(2 \pi)^{3}} \frac{g_{n}^{2}\left(p^{2}\right)}{k_{n}^{2}-p^{2}-\Pi^{(n)}(\sqrt{s}, \rho)+\mathrm{i} 0},
$$

where the integration on intermediate meson-baryon momenta is limited to a region $\Omega_{n}(\rho)$ ensuring that the intermediate nucleon energy is above the Fermi level in channels $n$ involving nucleons. The self-energy $\Pi^{(n)}(\sqrt{s}, \rho)$ stands for the sum of hadron self-energies in channel $n$. Of particular interest is the meson $(h)$ self-energy $\Pi_{h}^{(h N)}=\left(E_{N} / \sqrt{s}\right) \Pi_{h}$ in the diagonal $n \equiv(h N)$ channel, where the lab self-energy $\Pi_{h}$ is given by

$$
\Pi_{h}(\sqrt{s}, \rho) \equiv 2 \omega_{h} V_{h}=-\frac{\sqrt{s}}{E_{N}} 4 \pi F_{h N}(\sqrt{s}, \rho) \rho,
$$

depending implicitly on $\omega_{h}=m_{h}-B_{h}$ and on the off-shell two-body momenta $k, k^{\prime}$. This self-energy, once evaluated self-consistently while converting its $\sqrt{s}$ dependence into a full density dependence, serves as input to the Klein-Gordon bound-state equation

$$
\left[\nabla^{2}+\tilde{\omega}_{h}^{2}-m_{h}^{2}-\Pi_{h}\left(\omega_{h}, \rho\right)\right] \psi=0,
$$

in which $\tilde{\omega}_{h}=\omega_{h}-\mathrm{i} \Gamma_{h} / 2$, with $B_{h}$ and $\Gamma_{h}$ the binding energy and the width of the meson-nuclear bound state, respectively.

The resulting in-medium $K^{-} N$ isoscalar amplitudes above and below threshold are shown in Fig. 2. The real part of the amplitude is strongly attractive at subthreshold energies that according to the 
MESON 2014 $-13^{\text {th }}$ International Workshop on Production, Properties and Interaction of Mesons
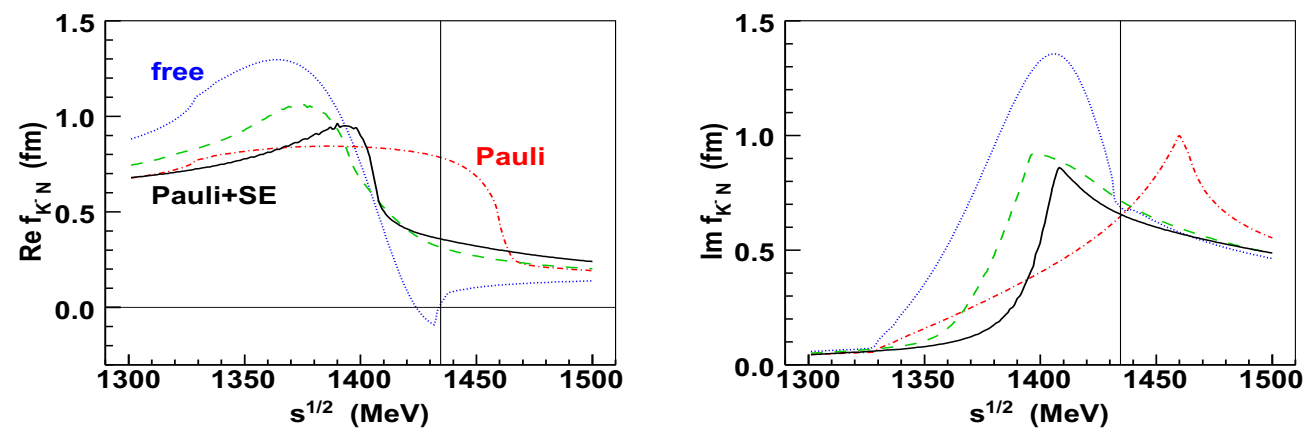

Figure 2. Near-threshold energy dependence of $\mathrm{K}^{-} N \mathrm{~cm}$ reduced scattering amplitudes (left: real, right: imaginary) in model NLO30 of Ref. [8] for free-space (dotted) and Pauli-blocked amplitudes at $\rho=\rho_{0}$ with (solid) and without (dot-dashed) meson and baryon self-energies (SE). The dashed curves show Pauli-blocked amplitudes with SE at $\rho=0.5 \rho_{0}$. The $K^{-} N$ threshold is marked by a thin vertical line.

discussion below are relevant to $K^{-}$atomic and nuclear states. The attraction as well as the imaginarypart absorptivity get moderately weaker for $\rho \geq 0.5 \rho_{0}$, as demonstrated by comparing on the left panel the solid curves $\left(\rho=\rho_{0}\right)$ with the dashed curves $\left(\rho=0.5 \rho_{0}\right)$. This implies that $K^{-}$bound states are very likely to exist, but with rather large widths generated by the imaginary-part absorptivity.

To determine the subthreshold energies for use in in-medium hadron-nucleon scattering amplitudes, we consider the downward energy shift $\delta \sqrt{s} \equiv \sqrt{s}-\sqrt{s_{\mathrm{th}}}$ where $\sqrt{s_{\mathrm{th}}} \equiv m_{h}+m_{N}$ and $s=\left(\sqrt{s_{\mathrm{th}}}-B_{h}-B_{N}\right)^{2}-\left(\vec{p}_{h}+\vec{p}_{N}\right)^{2}$, with $B_{h}$ and $B_{N}$ meson and nucleon binding energies. Since $\vec{p}_{h}+\vec{p}_{N} \neq 0$ in the meson-nuclear cm frame (approximately the lab frame), the associated negative contribution to $s$ has to be included. To leading order in binding energies and kinetic energies with respect to rest masses, and specializing to $\bar{K}$ mesons (denoted $h=K$ ), $\delta \sqrt{s}$ is expressed as

$$
\delta \sqrt{s} \approx-B_{N}-B_{K}-\xi_{N} \frac{p_{N}^{2}}{2 m_{N}}-\xi_{K} \frac{p_{K}^{2}}{2 m_{K}}, \quad \xi_{N(K)} \equiv \frac{m_{N(K)}}{m_{N}+m_{K}} .
$$

Using the Fermi Gas model for nucleons and the local density approximation for $\bar{K}$, assuming also the minimal substitution (MS) principle [17], one obtains

$$
\delta \sqrt{s} \approx-B_{N} \frac{\rho}{\bar{\rho}}-\xi_{N}\left[B_{K} \frac{\rho}{\rho_{0}}+T_{N}\left(\frac{\rho}{\bar{\rho}}\right)^{2 / 3}+V_{c}\left(\frac{\rho}{\rho_{0}}\right)^{1 / 3}\right]-\xi_{K} \frac{\sqrt{s}}{\omega_{K} E_{N}} 2 \pi \operatorname{Re} F_{\bar{K} N}(\sqrt{s}, \rho) \rho,
$$

where $V_{c}$ is the $K^{-}$Coulomb potential due to the finite-size nuclear charge distribution, $T_{N}=23.0$ $\mathrm{MeV}$ is the average nucleon kinetic energy, $B_{N} \approx 8.5 \mathrm{MeV}$ is an average nucleon binding energy and $\bar{\rho}$ and $\rho_{0}$ are the average nuclear density and nuclear-matter density, respectively. Expression (7) respects the low-density limit, $\delta \sqrt{s} \rightarrow 0$ upon $\rho \rightarrow 0$. For attractive scattering amplitudes, the last term of (7), in particular, provides substantial downward energy shift overlooked by many previous calculations that assumed $\vec{p}_{K}=0$ which is inappropriate for finite nuclei. Since $\sqrt{s}$ depends through Eq. (7) on $\operatorname{Re} F_{\bar{K} N}(\sqrt{s}, \rho)$ which by itself depends on $\sqrt{s}$, it is clear that for a given value of $B_{K}$, $F_{\bar{K} N}(\sqrt{s}, \rho)$ has to be determined self-consistently by iterating Eq. (7). This is done at each radial point where $\rho$ is given, and for each $B_{K}$ value during the calculation of bound states. The emerging correlation between the downward energy shift $\delta \sqrt{s}$ and the density $\rho$ renders $F_{\bar{K} N}(\sqrt{s}, \rho)$ into a 

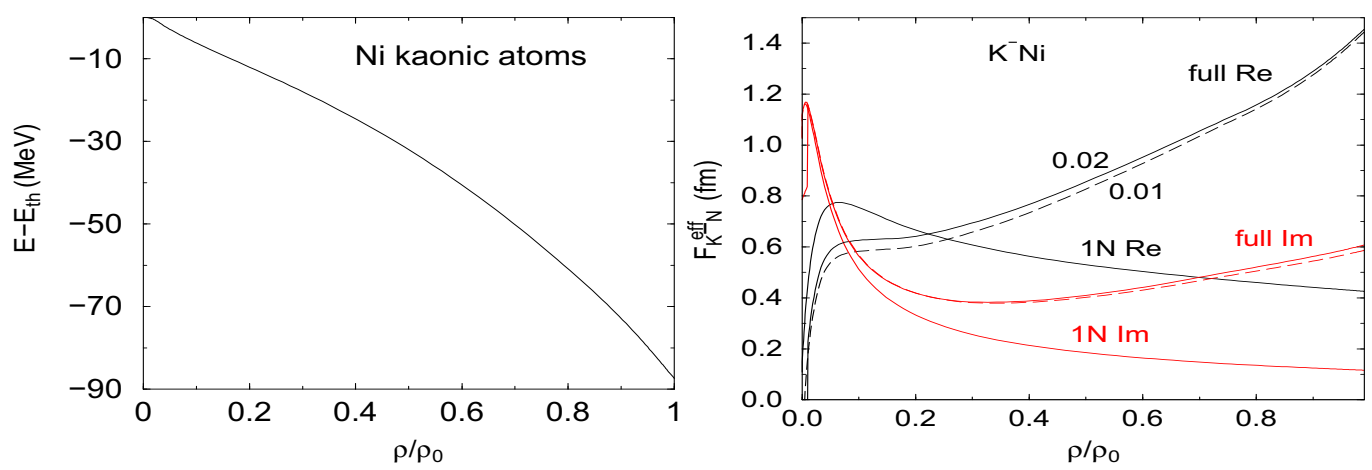

Figure 3. Left: subthreshold energies probed in $K^{-}-\mathrm{Ni}$ atom as a function of nuclear density, calculated selfconsistently within the IHW-based global fit to kaonic atoms using Eq. (7). Right: Kaonic-atom globally fitted amplitude $F_{K^{-}}^{\text {eff }}(\rho)$, marked "full", and the in-medium IHW-based amplitude $F_{K^{-} N}(\rho)$ in the absence of manynucleon contributions, marked " $1 \mathrm{~N}$ ", as a function of nuclear density in Ni [16]. Solid (dashed) curves are for matching to free-space amplitudes at $0.02(0.01) \rho_{0}$.

state-dependent function of the density $\rho$ alone, denoted for brevity by $F_{\bar{K} N}(\rho)$. This correlation is shown on the left side of Fig. 3 for kaonic atoms, where $B_{K^{-}} \approx 0$. The figure demonstrates appreciable energy shifts below threshold in kaonic atoms, although these are somewhat smaller (by $\sim 10 \mathrm{MeV}$ in $\mathrm{Ni}$ ) than the shifts evaluated in [16] without incorporating MS.

\section{$3 K^{-}$interactions in kaonic atoms}

The most recent kaonic-atom calculations are due to Friedman and Gal in Ref. [14], using inmedium $K^{-} N$ scattering amplitudes generated from the Cieplý-Smejkal (CS) NLO30 model as described in the previous section, and in Ref. [16] using Pauli blocked $K^{-} N$ scattering amplitudes generated from the Ikeda-Hyodo-Weise (IHW) NLO work. The CS [8] and IHW [7] free-space amplitudes $F_{K^{-} N}(\sqrt{s})$ agree semi-quantitatively with each other. The kaonic-atom fit in Ref. [16] considers in addition to the in-medium IHW-based one-nucleon (1N) amplitude $F_{K^{-} N}(\sqrt{s}, \rho)$ input also manynucleon absorptive and dispersive contributions, represented by energy-independent phenomenological amplitude $F_{K^{-} N}^{\text {many }}(\rho)$ with prescribed density dependence form that includes several fit parameters. The assumption of energy independence is motivated by observing that $K^{-}$absorption on two nucleons, which is expected to dominate $F_{K^{-} N}^{\text {many }}$, releases energy $\sim m_{K^{-}}$considerably larger than the subthreshold energies of less than $100 \mathrm{MeV}$ encountered in kaonic-atom calculations. The self-energy input $\Pi_{K^{-}}$to the KG equation (5) is now constructed from an effective $K^{-} N$ scattering amplitude $F_{K^{-} N}^{\text {eff }}=F_{K^{-} N}^{\text {one }}+F_{K^{-} N}^{\text {many }}$ which is iterated through the self-consistency expression (7). This introduces coupling between the many-nucleon fitted amplitude $F_{K^{-} N}^{\text {many }}$ and the converged one-nucleon amplitude $F_{K^{-} N}^{\text {one }}$ that evolves from the $1 \mathrm{~N}$ input amplitude $F_{K^{-} N}(\rho): F_{K^{-} N}^{\text {one }}(\rho) \rightarrow F_{K^{-} N}(\rho)$ upon $F_{K^{-N}}^{\text {many }} \rightarrow 0$.

The full effective amplitude $F_{K^{-} N}^{\text {eff }}(\rho)$ resulting from the global kaonic-atom fit in [16] is shown in the right panel of Fig. 3 marked "full", along with the in-medium IHW-based amplitude $F_{K^{-} N}(\rho)$ marked " $1 \mathrm{~N}$ ". The figure makes it clear that for densities exceeding $\sim 0.5 \rho_{0}$ the full effective amplitude departs appreciably from the in-medium IHW-based amplitude, which in the case of the imaginary part amounts to doubling the $1 \mathrm{~N}$ absorptivity of in-medium $K^{-}$mesons. For more details, see [16]. 
MESON 2014 $-13^{\text {th }}$ International Workshop on Production, Properties and Interaction of Mesons
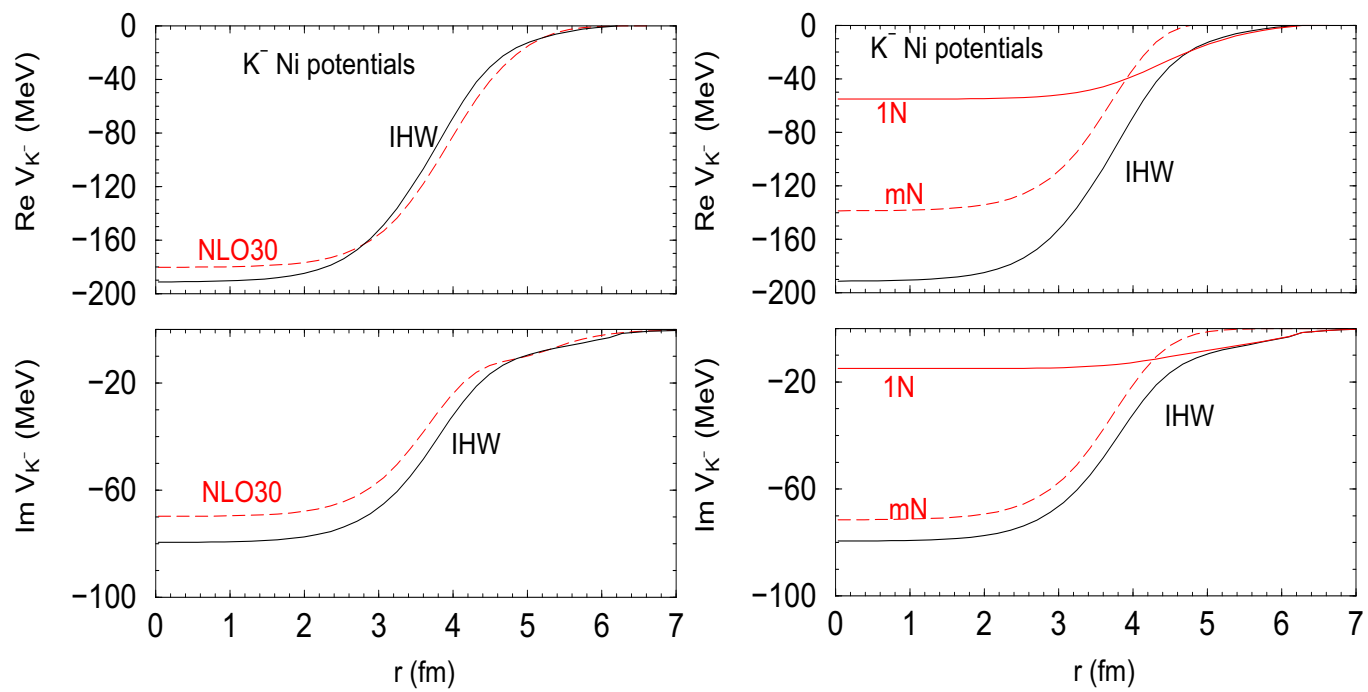

Figure 4. Left: Self-consistent $K^{-}$nuclear potentials $V_{K^{-}}$for $K^{-}$atoms of Ni derived from global fits based on in-medium IHW amplitudes [16], with the corresponding $1 \mathrm{~N}$ and many-nucleon $(\mathrm{mN})$ components on the right panel. The dashed curves in the left panel are derived from in-medium NLO30 amplitudes [14].

The $K^{-}$nuclear attraction and absorptivity deduced from global kaonic-atom fits are sizable at central nuclear densities. This is demonstrated for Ni in Fig. 4 by the real and imaginary parts of the potential $V_{K^{-}}$defined by (4). Although the potential depths might reflect merely a smooth extrapolation provided by the input components of the $K^{-} N$ amplitude, the potential at $0.5 \rho_{0}$ and perhaps up to $0.9 \rho_{0}$ is reliably determined in kaonic-atom fits [18]. It is reassuring that both IHW-based and NLO30-based fits agree with each other semi-quantitatively as shown on the left panel. The right panel of Fig. 4 shows a non-additive splitting of the fitted $K^{-}$-nuclear potential into a $1 \mathrm{~N}$ in-medium component, obtained on the assumption that there is no many-nucleon $(\mathrm{mN})$ component present, and a fitted $\mathrm{mN}$ component. The composition of the imaginary part of the potential is of particular interest, indicating that the $\mathrm{mN}$ component which is sizable in the nuclear interior becomes negligible about half a fermi outside of the half-density radius. This has implications for choosing optimally kaonicatom candidates where widths of two atomic levels can be measured so as to substantiate the $1 \mathrm{~N}$ vs $\mathrm{mN}$ pattern observed in global fits [19].

\section{Few-body kaonic quasibound states}

For $K^{-}$-nuclear three- and four-body calculations, a variant of the downward energy shift Eq. (7) derived for many-body calculations was formulated by Barnea, Gal and Liverts [13]:

$$
\delta \sqrt{s}=-\frac{B}{A}-\frac{A-1}{A} B_{K}-\xi_{N} \frac{A-1}{A}\left\langle T_{N: N}\right\rangle-\xi_{K}\left(\frac{A-1}{A}\right)^{2}\left\langle T_{K}\right\rangle,
$$

with $A$ the baryonic number, $B$ the total binding energy of the system, $B_{K}=-E_{K}, T_{K}$ the kaon kinetic energy operator in the total $\mathrm{cm}$ frame and $T_{N: N}$ the pairwise $N N$ kinetic energy operator in the $N N$ pair 
$\mathrm{cm}$ system. Note that $\delta \sqrt{s}$ is negative-definite by expression (8) which provides a self-consistency cycle upon requiring that $\sqrt{s}$ derived through Eq. (8) from the solution of the Schroedinger equation agrees with the value of $\sqrt{s}$ used for the input $V_{\bar{K} N}(\sqrt{s})$. Converged total binding energies calculated variationally in the hyperspherical basis are shown on the left panel of Fig. 5 for three- and four-body kaonic bound states. The corresponding $\bar{K} N \rightarrow \pi Y$ widths, calculated using the approximation

$$
\frac{\Gamma}{2} \approx\left\langle\Psi_{\text {g.s. }}\left|-\operatorname{Im} \mathcal{V}_{\bar{K} N}\right| \Psi_{\text {g.s. }}\right\rangle
$$

where $\mathcal{V}_{\bar{K} N}$ consists of all pairwise $\bar{K} N$ interactions, are plotted on the right panel as a function of $\delta \sqrt{s}$, with self-consistent values marked on each one of the $\Gamma$-vs- $\delta \sqrt{s}$ curves. Eq. (9) provides a good approximation owing to $\left|\operatorname{Im} \mathcal{V}_{\bar{K} N}\right| \ll\left|\operatorname{Re} \mathcal{V}_{\bar{K} N}\right|$ [20]. Expressions similar to (8) and (9) were used in $\bar{K} \bar{K} N N$ calculations. For details of the chirally-based energy-dependent $\bar{K} N$ interaction input and the actual calculations of these few-body kaonic clusters, see Ref. [13].
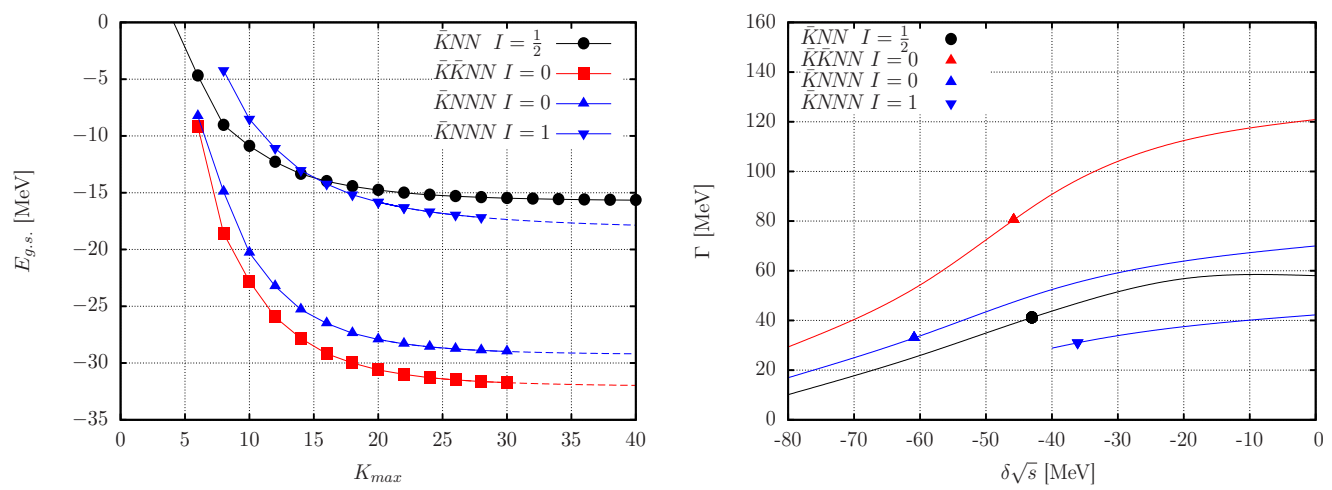

Figure 5. Left: binding energies of $\bar{K}$ and $\bar{K} \bar{K}$ few-body quasibound states from Ref. [13] calculated selfconsistently and plotted here as a function of the maximal total grand angular momentum $K_{\max }$ in the hyperspherical basis. Right: $\bar{K} N \rightarrow \pi Y$ widths (9) plotted as a function of $\delta \sqrt{s}$ (8).

With $\bar{K} N$ interactions that become weaker upon going subthreshold [20], the self-consistently calculated binding energies (widths) come out typically 10 (10-40) MeV lower than for threshold input interactions $V_{\bar{K} N}\left(\sqrt{s_{\mathrm{th}}}\right)$, as exhibited in the right panel by comparing the marked self-consistent values of $\Gamma$ to the threshold values $\Gamma(\delta \sqrt{s}=0)$, and in agreement with the recent Faddeev and FaddeevYakubovsky calculations by Maeda et al. [21] who used energy-independent interactions. In particular, the $I=1 / 2 \bar{K} N N$ g.s. known as ' $K^{-} p p$ ' is weakly bound, lying just $4.3 \mathrm{MeV}$ below the lowest threshold of $N+(\bar{K} N)_{I=0}$ at $-11.4 \mathrm{MeV}$. The latter value differs substantially from the $-27 \mathrm{MeV}$ assigned traditionally to the $\Lambda(1405)$ resonance and used in non-chiral calculations. Smaller differences occur in chiral models, where two $I=0$ poles appear, the upper of which is often identified with a $(\bar{K} N)_{I=0}$ quasibound state. For example, suppressing widths, the difference between the upper-pole positions in the IHW chiral model [7] (which is close to the one used in [13]) and in the recent RévaiShevchenko (RS) chirally motivated model [22] is $\Delta E(\bar{K} N)_{I=0}=7 \mathrm{MeV}$. A rough estimate of the model dependence expected for $K^{-} p p$ is $\Delta E\left(K^{-} p p\right) \approx 1.5 \Delta E(\bar{K} N)_{I=0}=10.5 \mathrm{MeV}$. Thus, a singlechannel $\bar{K} N N$ three-body calculation done $\grave{a}$ la Barnea et al. [13] using the RS model is expected to give $E\left(K^{-} p p\right) \approx-26 \mathrm{MeV}$. This has to be compared with $E=-32 \mathrm{MeV}$ obtained in the RS 
$\bar{K} N N-\pi \Sigma N$ coupled-channel Faddeev calculation [22]. The $6 \mathrm{MeV}$ missing in this crude estimate is of the order of magnitude expected [24] from upgrading a $\bar{K} N N$ single-channel calculation to a $\bar{K} N N-\pi \Sigma N$ coupled-channel calculation. In this respect we reject the unfounded criticism made by $\mathrm{RS}$ of the self-consistency procedure application in Ref. [13].

The widths exhibited in the figure, of order $40 \mathrm{MeV}$ for single- $\bar{K}$ clusters and twice that for double$\bar{K}$ clusters, are due to $\bar{K} N \rightarrow \pi Y$. Additional $\bar{K} N N \rightarrow Y N$ contributions of up to $\sim 10 \mathrm{MeV}$ in $K^{-} p p$ [23] (see, however, the estimate $\Gamma_{\bar{K} N N \rightarrow Y N} \sim 30 \mathrm{MeV}$ made in Ref. [25]) and $\sim 20 \mathrm{MeV}$ in the fourbody systems [13] are foreseen. Altogether, widths of order $50 \mathrm{MeV}$ or higher are anticipated for few-body kaonic quasibound states.

\section{Many-body kaonic quasibound states}

In-medium $\bar{K} N$ scattering amplitudes derived from the chirally motivated NLO30 model [8] were employed by Gazda and Mareš [15] to evaluate self-consistently $K^{-}$quasibound states across the periodic table, using static RMF nuclear-core densities. Calculated $K^{-}$binding energies and widths in $\mathrm{Ca}$ are listed in Table 1 for several choices of input interactions.

Table 1. Self-consistently calculated binding energies $B_{K}$ and widths $\Gamma_{K}$ (in $\mathrm{MeV}$ ) of $K^{-}$quasibound states in $\mathrm{Ca}$, using a static RMF Ca density and NLO30 in-medium $K^{-} N$ subthreshold amplitudes, see text.

\begin{tabular}{|l|cc|cc|cc|}
\hline & \multicolumn{2}{|c|}{ NLO30 } & \multicolumn{2}{c|}{$+p$ wave } & \multicolumn{2}{c|}{$+2 N$ abs. } \\
& $B_{K}$ & $\Gamma_{K}$ & $B_{K}$ & $\Gamma_{K}$ & $B_{K}$ & $\Gamma_{K}$ \\
\hline $1 s_{K}$ & 70.5 & 14.9 & 73.0 & 14.8 & 68.9 & 58.9 \\
$1 p_{K}$ & 50.6 & 18.0 & 53.1 & 17.9 & 49.2 & 53.6 \\
$1 d_{K}$ & 28.8 & 30.3 & 32.1 & 29.3 & 27.7 & 59.7 \\
$2 s_{K}$ & 23.9 & 33.8 & 26.3 & 34.2 & 21.6 & 67.1 \\
\hline
\end{tabular}

In addition to $B_{K}$ and $\Gamma_{K}$ values for NLO30 in-medium $s$-wave $K^{-} N$ interactions, we listed in Table 1 values derived (i) by adding a $\Sigma(1385)$-motivated $p$-wave $K^{-} N$ interaction from Ref. [26], thereby increasing $B_{K}$ marginally by a few $\mathrm{MeV}$ and modifying $\Gamma_{K}$ by less than $1 \mathrm{MeV}$, or (ii) by adding a two-nucleon $(2 N) K^{-} N N \rightarrow Y N$ absorption term estimated from fitting to kaonic atoms, resulting in $\lesssim 2 \mathrm{MeV}$ decrease of $B_{K}$ but substantially increasing the width to $\Gamma_{K} \sim(50-70) \mathrm{MeV}$. Given these large widths, it is unlikely that distinct quasibound states can be uniquely resolved, except perhaps in very light $K^{-}$nuclei.

The hierarchy of widths listed in Table 1 is also worth noting. With energy-independent potentials one expects maximal widths for the lowest, most localized $1 s_{K}$ states, and gradualy decreased widths in excited states which are less localized within the nucleus. The reverse is observed here, in particular upon excluding $2 \mathrm{~N}$ absorption. This is a corollary of requiring self consistency: the more excited a $K^{-}$quasibound state is, the lower nuclear density it feels, and a smaller downward shift into subthreshold energies it probes via the $\sqrt{s(\rho)}$ dependence. Since $\operatorname{Im} f_{K^{-} N}(\rho)$ decreases strongly upon going below threshold, see Fig. 2, its contribution to the calculated width gets larger, the higher the excited quasibound-state energy is.

\section{Summary and outlook}

In this overview of $\bar{K}$-nuclear bound-state calculations we have focused on the role played by the underlying meson-baryon subthreshold dynamics. It was shown how the energy dependence of the meson-baryon in-medium scattering amplitudes is converted into density dependence of the meson 
self-energies, or equivalently of meson-nucleus optical potentials. Based on global fits of $K^{-}$-atom data we argued that the in-medium chiral model input has to be supplemented by appreciable manynucleon dispersive and absorptive potential contributions which imply uniformly large widths of order $50 \mathrm{MeV}$ and more for $\bar{K}$-nuclear bound states. The experimental thrust at present and in the near future hinges on $K^{-} p p$ searches, as reviewed by Nagae in this conference [27].

\section{Acknowledgements}

This work was supported by the GACR Grant No. 203/12/2126, as well as by the EU initiative FP7, HadronPhysics3, under the SPHERE and LEANNIS cooperation programs.

\section{References}

[1] T. Hyodo, Nucl. Phys. A 914, 260 (2013)

[2] S. Wycech, Nucl. Phys. B 28, 541 (1971)

[3] W.A. Bardeen, E.W. Torigoe, Phys. Lett. B 38, 135 (1972)

[4] J.R. Rook, Nucl. Phys. A 249, 466 (1975)

[5] Z.-H. Guo, J.A. Oller, Phys. Rev. C 87, 035202 (2013)

[6] M. Bazzi et al. (SIDDHARTA Collaboration), Phys. Lett. B 704, 113 (2011), Nucl. Phys. A 881, 88 (2012)

[7] Y. Ikeda, T. Hyodo, W. Weise, Phys. Lett. B 706, 63 (2011), Nucl. Phys. A 881, 98 (2012)

[8] A. Cieplý, J. Smejkal, Nucl. Phys. A 881, 115 (2012)

[9] A. Gal, Nucl. Phys. A 914, 270 (2013)

[10] A. Gal, E. Friedman, N. Barnea, A. Cieplý, J. Mareš, D. Gazda, Acta Phys. Polon. B 45, 673 (2014)

[11] A. Cieplý, E. Friedman, A. Gal, D. Gazda, J. Mareš, Phys. Lett. B 702, 402 (2011), Phys. Rev. C 84, 045206 (2011)

[12] A. Cieplý, E. Friedman, A. Gal, V. Krejčiř́ík, Phys. Lett. B 698, 226 (2011)

[13] N. Barnea, A. Gal, E.Z. Liverts, Phys. Lett. B 712, 132 (2012)

[14] E. Friedman, A. Gal, Nucl. Phys. A 881, 150 (2012)

[15] D. Gazda, J. Mareš, Nucl. Phys. A 881, 159 (2012)

[16] E. Friedman, A. Gal, Nucl. Phys. A 899, 60 (2013)

[17] E. Friedman, A. Gal, Nucl. Phys. A 928, 128 (2014)

[18] N. Barnea, E. Friedman, Phys. Rev. C 75, 022202(R) (2007)

[19] E. Friedman, S. Okada, Nucl. Phys. A 915, 170 (2013)

[20] T. Hyodo, W. Weise, Phys. Rev. C 77, 035204 (2008)

[21] S. Maeda, Y. Akaishi, T. Yamazaki, Proc. Jpn. Acad. B 89, 418 (2013)

[22] J. Révai, N.V. Shevchenko, Phys. Rev. C 90, 034004 (2014)

[23] A. Doté, T. Hyodo, W. Weise, Nucl. Phys. A 804, 197 (2008), Phys. Rev. C 79, 014003 (2009)

[24] Y. Ikeda, T. Sato, Phys. Rev. C 79, 035201 (2009)

[25] M. Bayar, E. Oset, Phys. Rev. C 88, 044003 (2013)

[26] W. Weise, R. Härtle, Nucl. Phys. A 804, 173 (2008)

[27] T. Nagae, in these proceedings 